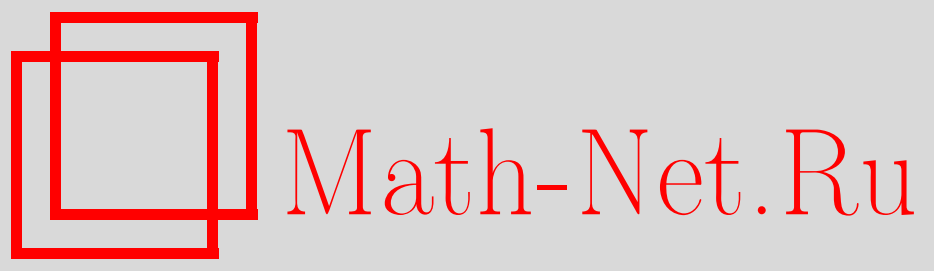

М. А. Манько, В. И. Манько, Квантовое преобразование Фурье и неравенства для томографической энтропии Реньи, ТМФ, 2009, том 160, номер 1, 143-156

DOI: https://doi.org/10.4213/tmf6386

Использование Общероссийского математического портала Math-Net.Ru подразумевает, что вы прочитали и согласны с пользовательским соглашением http://www . mathnet.ru/rus/agreement

Параметры загрузки:

IP: 52.6 .47 .48

26 апреля 2023 г., 18:03:06

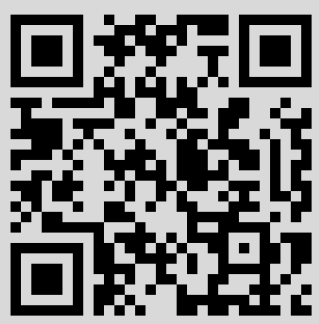




\title{
ФИЗИКА
}

Том 160, № 1

июль, 2009

\section{КВАНТОВОЕ ПРЕОБРАЗОВАНИЕ ФУРЬЕ И НЕРАВЕНСТВА ДЛЯ ТОМОГРАФИЧЕСКОЙ ЭНТРОПИИ РЕНЬИ}

\begin{abstract}
Показано, что энтропия Реньи, ассоциированная со спиновыми томограммами квантовых состояний, удовлетворяет новым неравенствам, зависящим от квантового преобразования Фурье. Получено предельное неравенство для энтропии фон Неймана спиновых квантовых состояний и введен новый вид энтропии, ассоциированный с квантовым преобразованием Фурье. Рассмотрены возможные связи с условиями субаддитивности и сильной субаддитивности для томографической энтропии и энтропии фон Неймана.
\end{abstract}

Ключевые слова: соотношения неопределенностей, энтропия, квантовая томография, квантовое преобразование Фурье.

\section{1. ВВЕДЕНИЕ}

В работе [1] был сформулирован подход к квантовой теории информации, основанный на введении так называемого вероятностного представления. При таком подходе элементы квантовой теории информации - кубиты, кудиты и операторы, описывающие состояния кудитов и связи между ними, - даны в виде функций, которые называются символами операторов. Для символов операторов правило умножения, называемое звездочным произведением, определяется некоторым интегральным нелокальным ядром [2]. Как для чистых, так и для смешанных состояний кудитов символы операторов плотности являются стандартными функциями распределения вероятностей. Поскольку состояния кудитов составных систем определяются стандартными распределениями вероятностей, все характеристики этих распределений, включая энтропию Шеннона [3] и энтропию Реньи [4], могут быть использованы для введения в квантовой теории информации энтропий, отвечающих символам операторов плотности, таких как, например, энтропия Реньи и относительная энтропия, определяемые символами соответствующих операторов. Энтропия Шеннона является предельным случаем энтропии Реньи, поэтому соответствующий аналог энтропии Шеннона, определяемой символом оператора плотности,

*Физический институт имени П. Н. Лебедева РАН, Москва, Россия.

E-mail: manko@sci.lebedev.ru 
и свойства этой энтропии могут быть получены в квантовой теории информации в рамках предложенного вероятностного представления.

Квантовой механике и квантовой теории информации свойственна некоторая фундаментальная особенность, отличающая их квантовую природу от природы их классических аналогов, - это соотношения неопределенностей. Соотношениям неопределенностей Гейзенберга [5], Шредингера [6] и Робертсона [7], [8], записанным для сопряженных переменных, таких как координаты и импульсы, соответствуют так называемые энтропийные соотношения неопределенностей. Энтропийные соотношения неопределенностей для непрерывных переменных были записаны в виде неравенств для энтропии Шеннона, связанной с плотностями вероятностей координаты и импульса, в работах [9]-[11].

В квантовой теории информации при подходе, основанном на вероятностном представлении, условия субаддитивности и сильной субаддитивности в работе [1] были получены для распределений вероятностей, описывающих квантовые состояния кудитов (так называемые спиновые томограммы), а также были прояснены некоторые соотношения между этими неравенствами и условиями субаддитивности и сильной субаддитивности. А именно, при подходе, основанном на вероятностном представлении, было построено томографическое отображение унитарной группы $U(n)$ в симплекс и с использованием этого отображения введены понятия энтропии Шеннона, энтропии Реньи и других видов энтропии для унитарной группы.

Энтропийные соотношения неопределенностей для конечномерных квантовых систем были получены в виде неравенств для энтропии Шеннона, связанной с распределениями вероятностей, отвечающих измерениям некоммутирующих наблюдаемых [12]-[16]. Соотношения неопределенностей для энтропии Реньи для распределений координаты и импульса, а также для конечномерных систем с измеряемыми наблюдаемыми, связанными квантовым преобразованием Фурье, были получены в работе [17]. Для систем с двумя и тремя подсистемами существуют известные неравенства для энтропии фон Неймана, которые называются соотношениями субаддитивности и сильной субаддитивности [18], [19].

Цель настоящей работы - обобщение энтропийных неравенств типа соотношений субаддитивности и сильной субаддитивности, полученных авторами в работе [1] в рамках вероятностного представления в квантовой теории информации, и нахождение новых энтропийных неравенств для спиновых томограмм, являющихся аналогами энтропийных неравенств, которые обсуждались в работах [12]-[17]. Для непрерывных переменных энтропийные неравенства для квантовых симплектических томограмм были рассмотрены в работах [20]-[25]. Существенным аспектом новых неравенств является их тесная связь со свойствами квантового преобразования Фурье, которая обсуждалась в работе [26] и использовалась в квантовой теории информации (см., например, [27]-[29]). Квантовое преобразование Фурье является важным элементом теории и играет ключевую роль в квантовых вычислениях и обработке квантовой информации.

Мы получим энтропийные неравенства, которые налагают некоторые ограничения на унитарные спиновые томограммы, связывая их с числом $\ln N$, где $N$ - раз- 
мерность гильбертова пространства. Число $\ln N$ появлялось в энтропийных неравенствах в работах [12]-[14], [16], но новым элементом настоящего исследования является непосредственная связь числа $\ln N$ с распределением томографической вероятности, определяющей состояние кудита в вероятностном представлении квантовой теории информации.

\section{2. СПИНОВЫЕ ТОМОГРАММЫ}

Пусть дано $N$-мерное гильбертово пространство состояний спиновой системы. Оно может быть представлено или как пространство состояний одной частицы со спином $j=(N-1) / 2$, или, в случае представления в виде произведения чисел $N=$ $n_{1} n_{2} \ldots n_{M}$, как пространство многочастичной спиновой системы с $j_{1}=\left(n_{1}-1\right) / 2$, $j_{2}=\left(n_{2}-1\right) / 2, \ldots, j_{M}=\left(n_{M}-1\right) / 2$.

$(N \times N)$-мерная матрица плотности $\rho$ квантового состояния может быть представлена в форме унитарной томограммы спинового состояния [30].

Томограмма спинового состояния с $j=(N-1) / 2$ определяется соотношением

$$
w(m, u)=\left\langle m\left|u^{\dagger} \rho u\right| m\right\rangle
$$

где $\rho$ - матрица плотности, $u$ - унитарная матрица размерности $N \times N, m=$ $-j,-j+1, \ldots, j-$ значения проекции спина на ось $z$. Томограмма $w(m, u)$ является неотрицательной функцией распределения вероятности случайной спиновой переменной, удовлетворяющей условию нормировки

$$
\sum_{m=-j}^{j} w(m, u)=1
$$

и равенству

$$
\int w(m, u) d u=1
$$

где $d u$ есть мера Хаара на унитарной группе, при этом $\int d u=1$. Важным свойством томограммы (1) является ее взаимосвязь с матрицей плотности $\rho$, а именно $\rho \leftrightarrow w(m, u)[1]$. Это означает, что квантовое состояние задано, если известна томограмма [31], [32].

\section{3. КВАНТОВОЕ ПРЕОБРАЗОВАНИЕ ФУРЬЕ}

Симметричная унитарная $(N \times N)$-матрица $F$ с матричными элементами $F_{j k}=$ $e^{2 \pi i j k / N} / \sqrt{N}, j, k=0,1, \ldots, N-1$, являющимися характерами неприводимого представления циклической группы $C_{N}$, может быть использована для обратимого преобразования нормированного комплексного вектора $\vec{a}$ с компонентами $a_{k}$ в комплексный вектор $\vec{a}^{(f)}$ с компонентами $a_{k}^{(f)}$ :

$$
a_{k}^{(f)}=\sum_{j=0}^{N-1} F_{k j} a_{j}, \quad a_{k}=\sum_{j=0}^{N-1}\left(F^{\dagger}\right)_{k j} a_{j}^{(f)} .
$$


Матрица $F$ с матричными элементами $F_{k j}$ удовлетворяет равенству

$$
F^{N}=1
$$

Преобразование (4) называется квантовым преобразованием Фурье для циклической группы $C_{N}$.

Если использовать для проекции спина индексы $m=-j,-j+1, \ldots, j$, оператор $\widehat{F}$ для квантового преобразования Фурье можно определить как

$$
\widehat{F}|m\rangle=\sum_{m^{\prime}=-j}^{j} F_{m^{\prime} m}\left|m^{\prime}\right\rangle,
$$

где симметричная матрица

$$
F_{m^{\prime} m}=\left\langle m^{\prime}|\widehat{F}| m\right\rangle
$$

имеет вид

$$
F_{m^{\prime} m}=\frac{1}{\sqrt{N}}\left(\begin{array}{ccccc}
1 & 1 & 1 & \ldots & 1 \\
1 & a & a^{2} & \ldots & a^{N-1} \\
1 & a^{2} & a^{4} & \ldots & a^{N-2} \\
\ldots & \ldots & \ldots & \ldots & \ldots \\
1 & a^{N-1} & a^{N-2} & \ldots & a
\end{array}\right), \quad a=e^{2 \pi i / N}
$$

Унитарный оператор квантового преобразования Фурье принимает вид

$$
\widehat{F}=\sum_{m=-j}^{j} \sum_{m^{\prime}=-j}^{j} F_{m^{\prime} m}\left|m^{\prime}\right\rangle\langle m| .
$$

В силу равенства $(5)$ имеем $(\widehat{F})^{N}=\hat{1}$, где $\hat{1}$ - единичный оператор.

\section{4. ТОМОГРАФИЧЕСКИЕ ЭНТРОПИИ ШЕННОНА И РЕНЬИ}

Используя стандартные определения теории вероятностей, можно ввести томографическую энтропию Шеннона [1], [3], [33] и томографическую энтропию Реньи [1], [4]. Томографическая энтропия Шеннона, определяемая томографическим символом оператора плотности, является функцией на унитарной группе

$$
H_{u}=-\sum_{m=-j}^{j} w(m, u) \ln w(m, u)
$$

Томографическая энтропия Реньи, определяемая томографическим символом оператора плотности, также является функцией на унитарной группе, но зависит от дополнительного параметра,

$$
R_{u}=\frac{1}{1-q} \ln \sum_{m=-j}^{j}(w(m, u))^{q} .
$$


В случае двух спиновых томограмм $w_{1}(m, u)$ и $w_{2}(m, u)$ мы определяем относительную $q$-энтропию, задаваемую томографическим символом оператора плотности, как

$$
\begin{gathered}
H_{q}\left(w_{1}(u) \mid w_{2}(u)\right)=-\sum_{m=-j}^{j} w_{1}(m, u) \ln _{q} \frac{w_{2}(m, u)}{w_{1}(m, u)}, \\
\ln _{q} x=\frac{x^{1-q}-1}{1-q}, \quad x>0, \quad q>0, \quad \ln _{q \rightarrow 1} x=\ln x .
\end{gathered}
$$

Относительная томографическая $q$-энтропия является неотрицательной функцией.

При $q \rightarrow 1$ имеем $R_{u} \rightarrow H_{u}$, и томографическая относительная $q$-энтропия преобразуется в относительную энтропию

$$
H\left(w_{1}(u) \mid w_{2}(u)\right)=-\sum_{m=-j}^{j} w_{1}(m, u) \ln \frac{w_{2}(m, u)}{w_{1}(m, u)} .
$$

Как показано в работе [1], минимальное значение на унитарной группе томографической энтропии Реньи равно квантовой энтропии Реньи:

$$
\min R_{u}=\frac{1}{1-q} \ln \operatorname{Tr} \rho^{q}
$$

Относительная энтропия (12) также является неотрицательной функцией для всех возможных значений параметра деформации $q$.

Минимальное значение энтропии $H_{u}$, задаваемое $(10)$ на унитарной группе, равно энтропии фон Неймана [1], [33]

$$
\min H_{u}=-\operatorname{Tr} \rho \ln \rho .
$$

\section{5. ЭНТРОПИЙНЫЕ НЕРАВЕНСТВА ШЕННОНА ПРИ ИЗМЕРЕНИИ НЕКОММУТИРУЮЩИХ НАБЛЮДАЕМЫХ}

Рассмотрим известные энтропийные неравенства [12]-[16], которые появляются в проблеме измерения двух наблюдаемых $\hat{A}$ и $\widehat{B}$ в конечномерном гильбертовом пространстве.

Пусть спектральные разложения эрмитовых операторов $\hat{A}$ и $\widehat{B}$ имеют вид

$$
\hat{A}=\sum_{k} A_{k}\left|a_{k}\right\rangle\left\langle a_{k}\left|, \quad \widehat{B}=\sum_{k} B_{k}\right| b_{k}\right\rangle\left\langle b_{k}\right|, \quad k=1, \ldots, N,
$$

где $A_{k}$ и $B_{k}$ - собственные значения наблюдаемых, $\left|a_{k}\right\rangle$ и $\left|b_{k}\right\rangle$ - их ортонормированные системы собственных векторов.

Для чистого состояния $|\psi\rangle$ имеются два распределения вероятностей

$$
p_{k}=\left|\left\langle a_{k} \mid \psi\right\rangle\right|^{2}, \quad q_{k}=\left|\left\langle b_{k} \mid \psi\right\rangle\right|^{2} .
$$

Соответствующие энтропии Шеннона, связанные с этими распределениями, имеют вид

$$
H_{p}=-\sum_{k} p_{k} \ln p_{k}, \quad H_{q}=-\sum_{k} q_{k} \ln q_{k},
$$


при этом они удовлетворяют неравенству, полученному в работе [34],

$$
H_{p}+H_{q} \geqslant-2 \ln \frac{1}{2}(1+c)
$$

где параметр с определяется максимальной величиной модуля скалярного произведения:

$$
c=\max _{j, k}\left|\left\langle a_{j} \mid b_{k}\right\rangle\right|
$$

Более сильное неравенство

$$
H_{p}+H_{q} \geqslant-2 \ln c
$$

было доказано в работе [12] (см. также [35], где была предпринята попытка его доказательства).

Для наблюдаемых $\hat{A}$ и $\widehat{B}$ с собственными векторами, имеющими взаимосвязанные базисы $\left|a_{k}\right\rangle$ и $\left|b_{k}\right\rangle[14]$, [26],

$$
\max \left|\left\langle a_{i} \mid b_{j}\right\rangle\right|=\frac{1}{\sqrt{N}},
$$

неравенство (22) принимает вид [14] $H_{p}+H_{q} \geqslant \ln N$. Таким образом, размерность гильбертова пространства $N$ появляется в энтропийном неравенстве.

Проблема взаимосвязанных базисов связана с геометрией конечных гильбертовых пространств [36], [37]. Она широко обсуждалась в связи с построением функции Вигнера для конечномерного гильбертова пространства, а также в квантовой криптографии [27], [38]-[41].

Энтропийные неравенства для энтропии Шеннона могут быть также получены при изучении проблемы измерения нескольких некоммутирующих наблюдаемых с ортонормированным набором собственных векторов, удовлетворяющих условию (23) (см. [14], [15]). В работе [13] энтропийные неравенства для энтропии Тсаллиса для непрерывных переменных были получены на базе неравенств Соболева, а в работе [17] аналогичные энтропийные соотношения неопределенностей для энтропии Реньи приведены для конечного гильбертова пространства и непрерывных переменных.

\section{6. ИЗВЕСТНЫЕ НЕРАВЕНСТВА ДЛЯ ДВУХЧАСТИЧНЫХ И ТРЕХЧАСТИЧНЫХ СИСТЕМ}

Энтропии, определяемые для символа оператора плотности, удовлетворяют некоторым известным неравенствам, полученным в работе [1]. Например, если спиновая система состоит из спинов $j_{1}$ и $j_{2}$, то базис в пространстве тензорного произведения состояний имеет вид

$$
\left|m_{1} m_{2}\right\rangle=\left|m_{1}\right\rangle\left|m_{2}\right\rangle .
$$

В этом случае томограмма является совместным распределением вероятностей двух случайных проекций спина $m_{1}=-j_{1},-j_{1}+1, \ldots, j_{1}$ и $m_{2}=-j_{2},-j_{2}+1, \ldots, j_{2}$, зависящим от унитарной матрицы $u$ размерности $\left(2 j_{1}+1\right)\left(2 j_{2}+1\right) \times\left(2 j_{1}+1\right)\left(2 j_{2}+1\right)$. Эта томограмма имеет вид

$$
w\left(m_{1}, m_{2}, u\right)=\left\langle m_{1} m_{2}\left|u^{\dagger} \rho(1,2) u\right| m_{1} m_{2}\right\rangle,
$$


где $\rho(1,2)$ - матрица плотности двухчастичной системы с матричными элементами

$$
\rho(1,2)_{m_{1} m_{2}, m_{1}^{\prime} m_{2}^{\prime}}=\left\langle m_{1} m_{2}|\rho(1,2)| m_{1}^{\prime} m_{2}^{\prime}\right\rangle
$$

Для томограммы можно ввести энтропию Шеннона

$$
H_{12}(u)=-\sum_{m_{1}=-j_{1}}^{j_{1}} \sum_{m_{2}=-j_{2}}^{j_{2}} w\left(m_{1}, m_{2}, u\right) \ln w\left(m_{1}, m_{2}, u\right)
$$

которая удовлетворяет условию субаддитивности для всех элементов унитарной группы

$$
H_{12}(u) \leqslant H_{1}(u)+H_{2}(u),
$$

где $H_{1}(u), H_{2}(u)$ - энтропии Шеннона, связанные с томограммами подсистем

$$
w_{1}\left(m_{1}, u\right)=\sum_{m_{2}=-j_{2}}^{j_{2}} w\left(m_{1}, m_{2}, u\right), \quad w_{2}\left(m_{2}, u\right)=\sum_{m_{1}=-j_{1}}^{j_{1}} w\left(m_{1}, m_{2}, u\right)
$$

соотношением

$$
H_{k}(u)=-\sum_{m_{k}=-j_{k}}^{j_{k}} w_{k}\left(m_{k}, u\right) \ln w_{k}\left(m_{k}, u\right), \quad k=1,2
$$

Из неравенства (28) в силу соотношения между энтропией фон Неймана и томографической энтропией следует известное неравенство [1], являющееся условием субаддитивности соответствующей энтропии фон Неймана для системы из двух подсистем

$$
S_{12} \leqslant S_{1}+S_{2}
$$

где

$$
S_{k}=-\operatorname{Tr} \rho_{k} \ln \rho_{k}, \quad k=1,2, \quad \rho_{1}=-\operatorname{Tr}_{2} \rho(1,2), \quad \rho_{2}=-\operatorname{Tr}_{1} \rho(1,2) .
$$

Для спиновой системы, состоящей из трех подсистем со спинами $j_{1}, j_{2}, j_{3}$ и матрицей плотности $\rho(1,2,3)$, спиновая томограмма может быть записана в виде

$$
w\left(m_{1}, m_{2}, m_{3}, u\right)=\left\langle m_{1} m_{2} m_{3}\left|u^{\dagger} \rho(1,2,3) u\right| m_{1} m_{2} m_{3}\right\rangle .
$$

С этой томограммой связана энтропия Шеннона $H_{123}(u)$, которая, в свою очередь, удовлетворяет условию сильной субаддитивности на унитарной группе [1]

$$
H_{123}(u)+H_{2}(u) \leqslant H_{12}(u)+H_{23}(u),
$$

где

$$
H_{123}(u)=-\sum_{m_{1}=-j_{1}}^{j_{1}} \sum_{m_{2}=-j_{2}}^{j_{2}} \sum_{m_{3}=-j_{3}}^{j_{3}} w\left(m_{1}, m_{2}, m_{3}, u\right) \ln w\left(m_{1}, m_{2}, m_{3}, u\right),
$$


при этом энтропии $H_{12}(u), H_{23}(u)$ и $H_{2}(u)$ определяются с помощью маргинальных томограмм

$$
\begin{aligned}
w_{12}\left(m_{1}, m_{2}, u\right) & =\sum_{m_{3}=-j_{3}}^{j_{3}} w\left(m_{1}, m_{2}, m_{3}, u\right), \\
w_{23}\left(m_{2}, m_{3}, u\right) & =\sum_{m_{1}=-j_{1}}^{j_{1}} w\left(m_{1}, m_{2}, m_{3}, u\right), \\
w_{2}\left(m_{2}, u\right) & =\sum_{m_{1}=-j_{1}}^{j_{1}} w_{12}\left(m_{1}, m_{2}, u\right) .
\end{aligned}
$$

Новое неравенство (33) не противоречит известному условию сильной субаддитивности для энтропии фон Неймана [18], [19]

$$
S_{123}+S_{2} \leqslant S_{12}+S_{23}, \quad S_{123}=-\operatorname{Tr} \rho_{123} \ln \rho_{123}
$$

а другие энтропии являются энтропиями фон Неймана для редуцированных матриц плотности $\rho(1,2)=\operatorname{Tr}_{3} \rho(1,2,3)$ и $\rho(2,3)=\operatorname{Tr}_{1} \rho(1,2,3)$.

Неравенства (28) и (33) являются новыми неравенствами для составных квантовых конечномерных систем, полученных в работе [1].

\section{7. НОВЫЕ НЕРАВЕНСТВА ДЛЯ ТОМОГРАФИЧЕСКОЙ ЭНТРОПИИ РЕНЬИ}

В этом разделе мы продолжим изучение томографических энтропий, следуя анализу, проделанному в работе [1], и выведем новые неравенства для спиновых томографических энтропий, связанных с квантовым преобразованием Фурье. Для непрерывных сопряженных переменных (координата и импульс) неравенства для энтропии Реньи, связанные с плотностями вероятностей по координате и импульсу, были выведены в работе [17]. Эти неравенства использовались при выводе новых интегральных неравенств для симплектической и оптической томограмм в работах [20], [22]-[25]. Аналог соотношения неопределенностей для энтропии Реньи для $N$-мерного гильбертова пространства был получен в работе [17] в виде

$$
\frac{1}{1-\alpha} \ln \sum_{k=1}^{N} \widetilde{p}_{k}^{\alpha}+\frac{1}{1-\beta} \ln \sum_{l=1}^{N} p_{l}^{\beta} \geqslant \ln N
$$

где

$$
\widetilde{p}_{k}=\left|\widetilde{a}_{k}\right|^{2}, \quad p_{l}=\left|a_{l}\right|^{2}, \quad \frac{1}{\alpha}+\frac{1}{\beta}=2,
$$

а комплексные числа $\widetilde{a}_{k}$ и $a_{l}$ связаны квантовым преобразованием Фурье

$$
\widetilde{a}_{k}=\frac{1}{\sqrt{N}} \sum_{l=1}^{N} \exp \left(\frac{2 \pi i k l}{N}\right) a_{l} .
$$


Ниже мы используем неравенства (36) для вывода новых неравенств для энтропии Шеннона и энтропии Реньи, определяемых унитарными спиновыми томограммами. Спиновая томограмма состояния с оператором плотности $\rho$ может быть представлена как столбец вектора вероятности на унитарной группе с компонентами $w_{m}(u)$. Введем другой $N$-вектор с компонентами $p_{m}(u)=\sqrt{w_{m}(u)}$. В силу неравенства $(36)$ для этих векторов и с учетом соотношения

$$
\left|\sum_{m^{\prime}=-j^{\prime}}^{j} F_{m m^{\prime}} \sqrt{w\left(m^{\prime}, u\right)}\right|=\sqrt{w_{F}(m, u)}
$$

где $F_{m m^{\prime}}$ задается в $(8)$ и $w_{F}(m, u)$ есть распределение вероятности, получаем неравенство

$$
\frac{1}{1-\alpha} \ln \sum_{m=-j}^{j} w(m, u)^{\alpha}+\frac{1}{1-\beta} \ln \sum_{m=-j}^{j} w_{F}(m, u)^{\beta} \geqslant \ln N .
$$

Используя также определение спиновой томограммы для чистого состояния $|\psi\rangle$, получаем другое аналогичное неравенство

$$
\frac{1}{1-\alpha} \ln \sum_{m=-j}^{j} w(m, u)^{\alpha}+\frac{1}{1-\beta} \ln \sum_{m=-j}^{j} w(m, F u)^{\beta} \geqslant \ln N,
$$

где $F$ - матрица квантового преобразования Фурье. Можно предположить, что неравенство (40) остается верным и для смешанного состояния.

Итак, для энтропии Реньи (11) мы имеем неравенство для каждой унитарной матрицы

$$
R_{\alpha}(u)+R_{\beta}(F u) \geqslant \ln N
$$

Унитарная спиновая томограмма частицы со спином $j$ для состояния с $(N \times N)$-матрицей плотности $\rho$, где $N=2 j+1$, должна удовлетворять неравенству (40). При $\alpha \rightarrow 1$ и $\beta \rightarrow 1$ получаем неравенства для энтропии Шеннона спинового состояния

$$
H(u)+H(F u) \geqslant \ln N
$$

Кроме того, справедливо неравенство

$$
H(u)+H_{F}(u) \geqslant \ln N,
$$

где $H_{F}(u)$ - энтропия Шеннона, связанная с распределением вероятности $w_{F}(m, u)$.

Для минимального значения энтропии Шеннона, достигаемого для унитарной матрицы $u_{0}$, имеется энтропия фон Неймана $H\left(u_{0}\right)=S_{\mathrm{vN}}$. Неравенство $(42)$, записанное для $u_{0}$,

$$
H\left(u_{0}\right)+H\left(F u_{0}\right) \geqslant \ln N
$$

дает неравенство для энтропии фон Неймана

$$
S_{\mathrm{vN}}+S\left(F u_{0}\right) \geqslant \ln N
$$


где $S\left(F u_{0}\right)$ - новая энтропия. Она имеет следующий физический смысл. Если оператор плотности квантового состояния спина задается в форме спектрального разложения

$$
\hat{\rho}=\sum_{q=-j}^{j} \lambda_{q}|q\rangle\langle q|,
$$

можно идентифицировать собственное состояние $|q\rangle$ оператора плотности $\hat{\rho}$ с "координатным" состоянием. При подходе со взаимосвязанными базисами и функцией Вигнера для конечного гильбертова пространства [26], [27], [38]-[42] состояния $|p\rangle=\widehat{F}|q\rangle$ с оператором преобразования Фурье $\widehat{F}$ интерпретируются как собственные состояния "импульса". Матричные элементы $\langle p|\widehat{F}| q\rangle=F_{p q}$ дают матрицу $F$, совпадающую с матрицей преобразования Фурье.

Итак, мы интерпретировали новое неравенство, как это было сделано в случае непрерывных переменных. Новая энтропия $S\left(F u_{0}\right)$ в $(45)$ является энтропией Шеннона для распределения “импульсов", если стандартная энтропия фон Неймана отождествляется с энтропией Шеннона для распределения "координат".

Рассмотрим пример состояния кубита с матрицей плотности

$$
\rho=\left(\begin{array}{ll}
1 & 0 \\
0 & 0
\end{array}\right)
$$

Оператор координаты $\hat{q}$ является $\sigma_{z}$-матрицей, и оператор импульса $\hat{p}$ является $\sigma_{x}$-матрицей. Собственными векторами оператора координаты $|q\rangle$ и оператора импульса $|p\rangle$ являются

$$
\left(\begin{array}{l}
1 \\
0
\end{array}\right), \quad\left(\begin{array}{l}
0 \\
1
\end{array}\right) \quad \text { и } \quad \frac{1}{\sqrt{2}}\left(\begin{array}{l}
1 \\
1
\end{array}\right), \quad \frac{1}{\sqrt{2}}\left(\begin{array}{r}
1 \\
-1
\end{array}\right) .
$$

соответственно. Матрица $F$ имеет вид

$$
F=\frac{1}{\sqrt{2}}\left(\begin{array}{rr}
1 & 1 \\
1 & -1
\end{array}\right)
$$

матрица $u_{0}=1$. Неравенство (45) обращается в равенство, так как $S_{\mathrm{vN}}=0, S(F)=$ $\ln 2, S_{\mathrm{vN}}+S(F)=\ln 2 \geqslant \ln 2$. Неравенство для энтропии Реньи также обращается в равенство

$$
R_{\alpha}\left(u_{0}\right)+R_{\beta}\left(F u_{0}\right)=\ln 2 \geqslant \ln 2 .
$$

В рассмотренном примере векторы $|q\rangle$ и $|p\rangle$ образуют так называемые взаимосвязанные базисы [39]-[42].

Следует заметить, что существуют энтропийные соотношения неопределенностей Шеннона для распределений, связанных с набором взаимосвязанных базисов [15], а также с парой ортогональных базисов [12]. Если взаимосвязанные базисы связаны квантовым преобразованием Фурье, неравенство (44) совпадает с полученным в работе [12].

В работе [1] мы ввели усредненные по группе энтропии Шеннона и Реньи. 
В силу инвариантности меры Хаара можно заключить, что усредненная по унитарной группе томографическая энтропия Шеннона удовлетворяет неравенству

$$
\bar{H}=\int H(u) d u \geqslant \frac{1}{2} \ln N .
$$

Также для усредненной по унитарной группе томографической энтропии Реньи (11) выполняется неравенство

$$
\bar{R}_{\alpha \beta}=\int R_{\alpha}(u) d u+\int R_{\beta}(u) d u \geqslant \ln N, \quad \frac{1}{\alpha}+\frac{1}{\beta}=2 .
$$

Чтобы проиллюстрировать полученные неравенства, рассмотрим смешанное состояние кубита с диагональной матрицей плотности

$$
\rho=\left(\begin{array}{ll}
a & 0 \\
0 & b
\end{array}\right), \quad a+b=1 .
$$

В этом случае неравенство (42) можно интерпретировать следующим образом. Энтропия фон Неймана этого состояния имеет вид

$$
S_{\mathrm{vN}}=-a \ln a-b \ln b .
$$

В результате квантового преобразования Фурье (48) матрица плотности принимает вид

$$
F^{\dagger} \rho F=\left(\begin{array}{cc}
1 / 2 & (a-b) / 2 \\
(a-b) / 2 & 1 / 2
\end{array}\right) .
$$

Соответствующая ей томографическая энтропия удовлетворяет равенству $H\left(F u_{0}\right)=$ $\ln 2, u_{0}=1$. Неравенство (42) принимает вид

$$
-a \ln a-b \ln b+\ln 2 \geqslant \ln 2,
$$

а это означает, что энтропия фон Неймана является неотрицательной величиной. Однако неравенство (43) дает лучшую оценку, поскольку число $\ln 2$ заменяется меньшим. Действительно, вектор томографической вероятности состояния кубита

$$
\vec{w}=\left(\begin{array}{l}
a \\
b
\end{array}\right)
$$

связан с амплитудой вектора вероятности с положительными компонентами

$$
\vec{W}=\left(\begin{array}{c}
\sqrt{a} \\
\sqrt{b}
\end{array}\right)
$$

В результате квантового преобразования Фурье этого вектора получается векторстолбец

$$
\vec{W}_{F}=\frac{1}{\sqrt{2}}\left(\begin{array}{c}
\sqrt{a}+\sqrt{b} \\
\sqrt{a}-\sqrt{b}
\end{array}\right) .
$$


Вектор распределения вероятности связан с рассмотренным выше вектором амплитуды вероятности

$$
\vec{w}_{F}=\left(\begin{array}{l}
1 / 2+\sqrt{a b} \\
1 / 2-\sqrt{a b}
\end{array}\right)
$$

Применяя неравенство, связывающее энтропии Шеннона для двух векторов (57) и (59), получаем

$$
-a \ln a-b \ln b-\left(\frac{1}{2}+\sqrt{a b}\right) \ln \left(\frac{1}{2}+\sqrt{a b}\right)-\left(\frac{1}{2}-\sqrt{a b}\right) \ln \left(\frac{1}{2}-\sqrt{a b}\right) \geqslant \ln 2
$$

или

$$
S_{\mathrm{vN}}-\left(\frac{1}{2}+\sqrt{a b}\right) \ln \left(\frac{1}{2}+\sqrt{a b}\right)-\left(\frac{1}{2}-\sqrt{a b}\right) \ln \left(\frac{1}{2}-\sqrt{a b}\right) \geqslant \ln 2 .
$$

Это неравенство не очевидно, хотя мы знаем, что энтропия фон Неймана $S_{\mathrm{vN}} \geqslant 0$.

Выведем некоторые неравенства для унитарной матрицы. Рассмотрим произвольную $(N \times N)$-унитарную матрицу $u_{j k}$. Справедливо неравенство

$$
-\sum_{j=1}^{N}\left(\left|u_{j k}\right|^{2} \ln \left|u_{j k}\right|^{2}+\left|(F u)_{j k}\right|^{2} \ln \left|(F u)_{j k}\right|^{2}\right) \geqslant \ln N
$$

или

$$
-\sum_{j=1}^{N} \sum_{k=1}^{N}\left(\left|u_{j k}\right|^{2} \ln \left|u_{j k}\right|^{2}+\left|(F u)_{j k}\right|^{2} \ln \left|(F u)_{j k}\right|^{2}\right) \geqslant N \ln N
$$

где $F_{j k}$ - матрица преобразования Фурье. Интегрируя неравенство (62) по унитарной группе с мерой Хаара с нормировкой (3), получаем неравенство

$$
-\int \sum_{j=1}^{N}\left|u_{j k}\right|^{2} \ln \left|u_{j k}\right|^{2} d u \geqslant \frac{1}{2} \ln N .
$$

На примере кубита мы показали, что для томограмм спиновых состояний, связанных квантовым преобразованием Фурье, имеют место условия в виде неравенств для томографических энтропий Шеннона. Аналогично можно получить неравенства и для томографических энтропий Реньи.

\section{8. ЗАКЛЮЧЕНИЕ}

Мы показали, что существуют несколько неравенств для энтропии Шеннона и энтропии Реньи, связанных с томограммами квантового спинового состояния. Эти неравенства дают новые соотношения для теории информации в дополнение к известным условиям субаддитивности и сильной субаддитивности. Физическое и информационное значения полученных неравенств требуют дальнейшего изучения. 
Благодарности. Инициатором этих исследований был профессор Р. Вилела Мендеш, и плодотворные дискуссии с ним оказали значительную помощь авторам при получении результатов, представленных в настоящей статье. Авторы выражают ему искреннюю благодарность. Работа поддержана РФФИ (грант № 07-02-00598). Авторы благодарят Университет Лиссабона, где эта работа была частично выполнена. М. А. Манько благодарит организаторов Международной конференции "Нелинейная физика. Теория и эксперимент. V" за гостеприимство и РФФИ за финансовую поддержку (грант № 08-02-08174).

\section{Список литературы}

[1] M. A. Man'ko, V. I. Man'ko, R. Vilela Mendes, J. Russ. Laser Res., 27:6 (2006), 507-532.

[2] O. V. Man'ko, V. I. Man'ko, G. Marmo, J. Phys. A, 35:3 (2002), 699-719.

[3] C. E. Shannon, Bell System Tech. J., 27 (1948), 379-423.

[4] A. Rényi, Probability Theory, North-Holland Ser. Appl. Math. Mech., 10, Amsterdam, North-Holland, 1970.

[5] W. Heisenberg, Z. Phys., 43:3-4 (1927), 172-198.

[6] Э. Шредингер, "K принципу неопределенности Гейзенберга", Избранные труды по квантовой механике, Наука, М., 1976, 210-217.

[7] H. P. Robertson, "A general formulation of the uncertainty principle and its classical interpretation", Proc. American Phys. Soc. Abstracts, Phys. Rev., 35 (1930), 667.

[8] H. P. Robertson, Phys. Rev., 46:9 (1934), 794-801.

[9] I. I. Hirschman Jr., Amer. J. Math., 79:1 (1957), 152-156.

[10] I. Białynicki-Birula, J. Mycielski, Comm. Math. Phys., 44:2 (1975), 129-132.

[11] В.В. Додонов, В.И. Манько, Инварианты и эволюиия нестационарных квантовых систем, Труды ФИАН, 183, ред. М. А. Марков, Наука, М., 1987.

[12] H. Maassen, J. B. M. Uffink, Phys. Rev. Lett., 60:12 (1988), 1103-1106.

[13] A. K. Rajagopal, Phys. Lett. A, 205:1 (1995), 32-36.

[14] J. Sánchez-Ruiz, Phys. Lett. A, 201:2-3 (1995), 125-131.

[15] M. A. Ballester, S. Wehner, Phys. Rev. A, 75:2 (2007), 022319.

[16] A. Azarchs, Entropic uncertainty relations for incomplete sets of mutually unbiased observables, arXiv: quant-ph/0412083.

[17] I. Bialynicki-Birula, Phys. Rev. A, 74:5 (2006), 052101.

[18] E. H. Lieb, M. B. Ruskai, J. Math. Phys., 14:2 (1973), 1938-1941.

[19] M. B. Ruskai, Int. J. Quant. Inform., 3:3 (2005), 579-590; Erratum, 4:4 (2006), 747-748; arXiv: quant-ph/0404126.

[20] S. De Nicola, R. Fedele, M. A. Man'ko, V. I. Man'ko, Eur. Phys. J. B, 52:2 (2006), 191-198.

[21] M. A. Man'ko, J. Russ. Laser Res., 27:5 (2006), 405-413.

[22] M. A. Man'ko, V. I. Man'ko, S. De Nicola, R. Fedele, Acta Phys. Hung. B, 26:1-2 (2006), $71-77$.

[23] С. Де Никола, Р. Феделе, М. А. Манько, В. И. Манько, ТМФ, 152:2 (2007), 241-247.

[24] M. A. Man'ko, "Tomographic entropy and new entropic uncertainty relations", Quantum Theory: Reconsideration of Foundations - 4 (Växjö, Sweden, 2007), AIP Conf. Proc., 962, eds. G. Adenier, A. Yu. Khrennikov, P. Lahti, V. I. Man'ko, T. Nieuwenhuizen, AIP, Melville, NY, 2007, 132-139.

[25] S. De Nicola, R. Fedele, M. A. Man'ko, V.I. Man'ko, J. Phys. Conf. Ser., 70:1 (2007), 012007.

[26] J. Schwinger, Proc. Natl. Acad. Sci. USA, 46:4 (1960), 570-579; Quantum Kinematics and Dynamics, Benjamin, New York, 1970.

[27] S. Zhang, C. Lei, A. Vourdas, J. A. Dunningham, J. Phys. B, 39 (2006), 1625-1637. 
[28] P.W. Shor, SIAM J. Sci. Statist. Comput., 26:5 (1997), 1484-1509; arXiv: quant-ph/9508027.

[29] Y. S. Weinstein, M. A. Pravia, E. M. Fortunato, S. Lloyd, D. G. Cory, Phys. Rev. Lett., 86:9 (2001), 1889-1891.

[30] V. I. Man'ko, G. Marmo, E. C. G. Sudarshan, F. Zaccaria, Phys. Lett. A, 327:5-6 (2004), $353-364$.

[31] V. V. Dodonov, V. I. Man'ko, Phys. Lett. A, 229:6 (1997), 335-339.

[32] В. И. Манько, О. В. Манько, ЖЭТФ, 112:3 (1997), 796-804.

[33] O. V. Man'ko, V. I. Man'ko, J. Russ. Laser Res., 25:2 (2004), 115-122.

[34] D. Deutsch, Phys. Rev. Lett., 50:9 (1983), 631-633.

[35] K. Kraus, Phys. Rev. D, 35:10 (1987), 3070-3075.

[36] W. K. Wootters, Ann. Phys., 176:1 (1987), 1-21.

[37] W. K. Wootters, Picturing qubits in phase space, arXiv: quant-ph/0306135.

[38] M. R. Kibler, Collect. Czech. Chem. Commun., 70 (2005), 771.

[39] M. Planat, Internat. J. Modern Phys. B, 20:11-13 (2006), 1833-1850; arXiv: math-ph/0510044.

[40] A. B. Klimov, C. Muñoz, J. L. Romero, J. Phys. A, 39:46 (2006), 14471-14497.

[41] C. Cormick, E. F. Galvão, D. Gottesman, J.P. Paz, A. O. Pittenger, Phys. Rev. A, 73:1 (2006), 012301; arXiv: quant-ph/0506222.

[42] M. Planat, H. Rosu, Eur. Phys. J. D, 36:1 (2005), 133-139. 